\title{
Nachruf Prof. Dr. med. Georg Peters
}

๑) Springer-Verlag GmbH Germany, part of Springer Nature 2018

Mit tiefer Betroffenheit haben wir vom Tod von Prof. Dr. med. Georg Peters erfahren.

Georg Peters war ein führender Experte auf dem Gebiet der Infektionsmedizin und gehörte zu einer kleinen Gruppe von Wissenschaftlern, die früh Defizite in der Versorgung von Infektionspatienten in Deutschland erkannt und deshalb einen Ausbau der Klinischen Infektiologie gefordert haben. Er war maßgeblich und aktiv an der Schaffung eines Förderprogramms beteiligt, das zur Etablierung von mehreren Lehrstühlen und Klinischen Forschergruppen in der klinischen Infektiologie an deutschen Universitäten geführt hat. Georg Peters hat immer die Notwendigkeit einer guten Ko-operation von Mikrobiologen, Hygienikern und klinischen Infektiologen gesehen und ist nie müde geworden, dies zu propagieren und einzufordern. Seinem großen Engagement haben wir es zu verdanken, dass die Infektiologie heute als ein wichtiges wissenschaftliches und klinisches Fach angesehen wird, das weiter ausgebaut werden muss. Noch kurz vor seinem Tod hat er eine Initiative ergriffen, die der weiteren Etablierung der Infektiologie in Deutschland dienen sollte.

Mit Georg Peters verliert die Infektiologie in Deutschland einen ihrer profiliertesten Vertreter und einen ihrer stärksten Förderer. Die Deutsche Gesellschaft für Infektiologie trauert um ihr langjähriges Mitglied, das die Entwicklung unseres Faches über viele Jahre entscheidend gefördert hat. Seine Unterstützung und seine kritische Begleitung werden uns sehr fehlen. Wir erinnern uns an einen hervorragenden Wissenschaftler, einen klugen Ratgeber und an einen ganz besonderen Menschen.

Prof. Dr. Gerd Fätkenheuer (Präsident)

Deutsche Gesellschaft für Infektiologie (DGI) 